\title{
Pegylation of poly( $\gamma$-benzyl-L-glutamate) nanoparticles is efficient for avoiding mononuclear phagocyte system capture in rats
}

This article was published in the following Dove Press journal:

International Journal of Nanomedicine

8 December 2010

Number of times this article has been viewed

Ipek Özcan'

Freimar Segura-Sánchez ${ }^{2,3}$

Kawthar Bouchemal ${ }^{2}$

Murat Sezak ${ }^{4}$

Özgen Özer ${ }^{1}$

Tamer Güneri'

Gilles Ponchel ${ }^{2}$

'Ege University, Faculty of Pharmacy, Department of Pharmaceutical

Technology, Izmir, Turkey; ${ }^{2}$ Université Paris Sud-I I, Faculty of Pharmacy,

Physicochimie Pharmacotechnie Biopharmacie, Chatenay-Malabry,

France; ${ }^{3}$ Universidad de Antioquia, Facultad de Química Farmacéutica, Departamento de Farmacia, Medellín, Colombia; ${ }^{4}$ Ege University, Faculty of Medicine, Department of Pathology, Izmir, Turkey
Correspondence: Ipek Ozcan Ege University, Faculty of Pharmacy, Department of Pharmaceutical Technology, 35100 Izmir, Turkey $\mathrm{Tel}+902323884000 / / 368$

$\mathrm{Fax}+902323885258$

Email ipek.ozcan@ege.edu.tr
Abstract: Poly( $\gamma$-benzyl-L-glutamate) (PBLG) derivatives are synthetic polypeptides for preparing nanoparticles with well controlled surface properties. The aim of this paper was to investigate the biodistribution of pegylated PBLG in rats. For this purpose, nanoparticles were prepared by a nanoprecipitation method using mixtures of different PBLG derivates, including a pegylated derivate to avoid mononuclear phagocyte system uptake. The morphology, size distribution, and surface charge of the nanoparticles were investigated as a function of the amount of polymer employed for the preparation. Moderately polydispersed nanoparticles (polydispersity index less than 0.2) were obtained. Their size increased with polymer concentration. The zeta potential values were negative whatever the formulations. The availability of polyethylene glycol chains on the nanoparticles' surface was confirmed by measuring the decrease in bovine serum albumin adsorption. For in vivo distribution studies, pegylated and nonpegylated nanoparticles were prepared with polymer mixtures containing PBLG-fluorescein isothiocyanate and imaged by fluorescence microscopy to measure their accumulation in liver and spleen tissues of rats after intravenous administration. Injection of stealth formulations resulted in negligible fluorescence in liver and spleen compared with nonpegylated formulations, which suggests that these nanoparticles are promising candidates as a stealth-type long-circulating drug carrier system and could be useful for active targeting of drugs while reducing systemic side effects.

Keywords: PBLG, physicochemical characterization, biodistribution, nanoparticles, pegylation

\section{Introduction}

Synthetic polypeptides are potential carriers for the delivery of drugs, owing to their low toxicity, biocompatibility, biodegradability, adjustable structures, and well controlled dimensions. Polypeptides can be conveniently synthesized using $\mathrm{N}$-carboxyanhydrides (NCAs) by ring-opening polymerization, which utilizes a wide variety of monomers containing various functional side groups. Similar to natural polypeptides, these polymers possess the ability to self-assemble into well defined, ordered structures. The use of polypeptidic polymers in the preparation of nanoparticles for drug delivery and targeting is an attractive area in nanomedicine..$^{1-3}$ Poly $(\gamma$-benzyl-L-glutamate) (PBLG), a synthetic and biodegradable polypeptide, has attracted attention for use in biomedical applications because various chemical moieties could be quite easily introduced into the structure of PBLG to form various copolymers. ${ }^{2}$ The functional side group of carboxyl (-COOH) in glutamic acid units can be modified by chemical reactions to form new molecular structures. ${ }^{4,5}$ As an alternative, copolymers can be obtained by initiating the polymer synthesis with a molecule of biological interest, such as a polyethylene glycol (PEG) chain or a cyclodextrin moiety. ${ }^{2}$ Further, it has 
been demonstrated that multifunctionalized nanoparticles exhibiting interesting surface properties could be obtained by preparing nanoparticles from mixtures of these different derivates.

Injectable polymeric nanoparticles have received much attention. Their use can be seen to prolong the blood halflife of drugs and increase their efficacy. ${ }^{6}$ Alternatively, they represent very attractive properties for targeting applications for improving the selectivity in drug delivery or for imaging purposes. These latter applications necessitate an efficient control of their body distribution. Such a distribution after intravenous (IV) injection is considerably influenced by their interactions with the biological environment and their physicochemical properties, including particle size, surface charge, morphology, and surface hydrophilicity. ${ }^{7-9}$ Their size can affect the biodistribution of intravascularly injected nanoparticles. Studies have shown that large nanoparticles are sequestered in the liver, consistent with observations in the spleen, whereas very small nanoparticles (less than $100 \mathrm{~nm}$ ) can pass through the sinusoidal fenestrations in the liver and be entrapped by underlying parenchymal cells. ${ }^{10}$ The size of polymeric nanoparticles can be adjusted to some extent as it depends on the polymer type, molecular weight, and concentration. Thus, to some extent, convenient particle size can be looked for by adjusting the polymer properties. ${ }^{11-13}$

Further, the potential of injectable polymeric nanoparticles is compromised by their rapid elimination from the bloodstream and their accumulation in the tissues of the mononuclear phagocyte system (MPS), mainly the Kupffer cells in the liver and the spleen macrophages. ${ }^{14-16}$ This prevents their application in controlled drug delivery and drug targeting to tissues other than the MPS.

The preferred method for the preparation of long-circulating nanoparticles is to modify their surface by adding hydrophilic polymers. PEG has been widely used for modifying the surface of particles, leading to stealth-type (long-circulating) carriers. ${ }^{17-19}$ Pegylation simply refers to the decoration of a particle surface by PEG chains, which can be obtained through different methods such as physical adsorption, covalent grafting, or the self-assembling of PEG copolymers. ${ }^{20-22}$ These PEG chains create a hydrophilic layer in the surface of the particles, which acts as a barrier that is able to block the adhesion of opsonins present in the blood serum so that the particles can remain camouflaged or invisible to phagocytic cells. ${ }^{15}$ Many different types of PEG-containing polymers have been tested for their ability to impart stealth characteristics to polymeric nanoparticles. Particularly, poly(lactide-coglycolide) (PLGA)-PEG and poly(lactide) (PLA)-PEG nanoparticles have been extensively investigated for their potential as controlled and targeted drug delivery systems. ${ }^{23,24}$ It has been shown that the IV administration of these nanoparticles loaded with a drug in experimental animals results in prolonged drug residence in systemic blood circulation. ${ }^{25}$ For example, after IV administration, PLA-PEG and PLGA-PEG nanoparticles remain in the systemic circulation for days, whereas PLA and PLGA nanoparticles are removed from blood within a few minutes. ${ }^{26}$

Therefore, when conceiving original nanoparticles for targeting applications, it is not only necessary to adjust their morphology, generally looking for small particles, but also to prevent the opsonization phenomenon. Obviously, pegylation remains nowadays a very attractive strategy for achieving this goal. The aim of this study was to investigate the biodistribution and efficacy of the pegylation of recently developed multifunctional PBLG nanoparticles. ${ }^{2,27,28}$ For this purpose, nanoparticles were prepared by a nanoprecipitation method using mixtures of different PBLG derivates, including a pegylated derivate, to avoid MPS uptake. Further, their distribution in tissues were investigated using fluorescein isothiocyanate (FITC) derivate-tagged nanoparticles after IV administration in rats, with a specific emphasis on their affinity for liver and spleen tissues, known as the major organs involved in the capture of nanoparticles in vivo.

\section{Materials and methods \\ Materials}

N,N-dimethylformamide (DMF; Acros, Belgium, 99\%) and benzylamine (Janssen Chimica, Belgium) were distilled under reduced pressure over $\mathrm{BaO}$ and $\mathrm{KOH}$, respectively, and stored under argon atmosphere. $\gamma$-benzyl-L-glutamate NCA, from ISOCHEM-SNPE (France), was used as received. Methoxy poly (ethylene glycol)-amine (mPEG-NH${ }_{2}$ ), $\mathrm{Mw}=5000 \mathrm{gmol}^{-1}$ from Shearwater Corporation (USA) was dried separately under vacuum over $\mathrm{P}_{2} \mathrm{O}_{5}$ at $30^{\circ} \mathrm{C}$ for 24 hours. Benzylamine (Bnz) and FITC were purchased from Sigma-Aldrich (Germany). Water was purified by reverse osmosis (Milli-Q, Millipore, USA). All other solvents and chemicals used were of analytical grade.

\section{Synthesis of PBLG derivatives}

Three different PBLG derivatives (PBLG-Bnz, PBLG-PEG, and PBLG-FITC) were synthesized by anionic ring-opening polymerization of NCA initiated by Bnz, mPEG-NH2, or FITC, respectively, in DMF. 2,29 To sum up, $\mathrm{N}$ millimoles of NCA were weighed under argon atmosphere in a degassed, three-necked, round-bottomed flask equipped with a 
thermometer, mechanical stirrer, and refrigerant with a silica gel guard and a bubble detector. NCA was dissolved in DMF at room temperature under mechanical stirring and argon flux. After about 10 minutes, the initiator solution was added and $\mathrm{CO}_{2}$ emission was observed. The reactions were conducted under argon atmosphere, and mixtures were stirred at $30^{\circ} \mathrm{C}$ until the characteristic Fourier transform infrared (FT-IR) NCA bands disappeared from the spectrum (Perkin-Elmer 1750, Norwalk, USA). Further, the mixture was precipitated in an excess of cold diethyl ether. The precipitates were filtered, washed with diethyl ether, and finally dried under vacuum at $35^{\circ} \mathrm{C}$ for at least 12 hours. Polymer molecular weights were characterized by Ubbelohde viscometer (Schott Geraete GmbH, Germany) using the Mark-Houwink equation $\left([\eta]=1.58 \times 10^{-5} \mathrm{Mw}^{1.35}\right)$; intrinsic viscosity $(\eta)$ was measured at $25^{\circ} \mathrm{C}$. Furthermore, both copolymers (PBLGBnz and PBLG-PEG) were dissolved in deuteriochloroform $\left(\mathrm{CDCl}_{3}\right)$ in nuclear magnetic resonance (NMR) tubes, and ${ }^{1} \mathrm{H}-\mathrm{NMR}$ spectras were recorded with a Bruker Advance (Bruker, Karlsruhe, Germany) spectrometer operated at $400 \mathrm{MHz}$.

\section{Preparation of nanoparticles}

Nanoparticles were prepared with the different PBLG derivatives by a modified nanoprecipitation method either using pure copolymers or a mixture of the different copolymers. ${ }^{27}$ FITC-labeled nanoparticles were prepared with PBLG-Bnz:PBLG-FITC and PBLG-PEG:PBLG-FITC polymer mixtures at the $80: 20$ ratio. Briefly, copolymers or copolymer mixtures were dissolved in $5 \mathrm{~mL}$ of tetrahydrofurane at $30^{\circ} \mathrm{C}$ using different amounts $(5,10,15,20,25 \mathrm{mg})$. This organic solution was added to $10 \mathrm{~mL}$ of Milli-Q water by dripping, under magnetic stirring, without the presence of any surfactant. The mixture was then transferred to a Teflon flask. The solvent was gently evaporated, at $30^{\circ} \mathrm{C}$, under a light air flow. Nanoparticles were washed with $5 \mathrm{~mL}$ of Milli-Q water, and finally the suspension was partly evaporated to yield $10 \mathrm{~mL}$ of nanoparticle suspension. The suspensions were kept at $+4^{\circ} \mathrm{C}$ in capped glass vials before use. Furthermore, their stability under these conditions was checked on a 4-week period during which the samples were visually inspected and their physicochemical characteristics regularly measured.

\section{Physicochemical characterization of nanoparticles}

The average particle size and size polydispersity of the nanoparticles were determined by dynamic laser light scattering (Nanosizer Coulter N4 Plus ${ }^{\circledR}$, Malvern Instruments, Malvern, UK). The $\zeta$ potential of the nanoparticles was measured in aqueous dispersion with a Zetasizer 4 (Malvern Instruments) at $25^{\circ} \mathrm{C}$. Experimental values were the average of six different preparations. Further, nanoparticles were imaged by transmission electron microscopy (TEM) (TEM-Philips EM 208, Orsay, France) operating at $80 \mathrm{kV}$, and images were acquired using a high-resolution camera (Advantage HR3/12GO4, AMTHamamatsu, France).

\section{Isothermal titration calorimetry (ITC) experiments}

In accordance with a previously published method, an isothermal calorimeter titration apparatus (MicroCal Inc., USA) has been used to evaluate the capacity of nanoparticles to avoid protein adsorption and therefore to confirm the presence of PEG chains at the surface of the nanoparticles. ${ }^{2}$ Bovine serum albumin (BSA) was used, as it has a major representative globular protein present in the serum and is known for being easily adsorbed on polymeric surfaces. The ITC instrument was periodically calibrated either electrically using an internal electric heater or chemically by measuring the dilution enthalpy of methanol in water. This standard reaction was in agreement $(1 \%-2 \%)$ with MicroCal constructor data. In a typical experiment, aliquots of $10 \mu \mathrm{L}$ of a BSA solution $\left(5.4 \times 10^{-2} \mathrm{mM}\right)$ filled into a syringe containing $283 \mu \mathrm{L}$ were used to titrate a suspension of nanoparticles (pegylated or nonpegylated) $\left(2.7 \times 10^{-2} \mathrm{mM}\right)$ into the calorimetric cell and accurately thermostated at $37^{\circ} \mathrm{C}(310 \mathrm{~K})$. Intervals between injections were 300 seconds, and agitation speed was $270 \mathrm{rpm}$. A control experiment was also performed, which consisted of successive injections of a BSA solution in the measuring cell filled with Milli-Q water. The corresponding heat flow was recorded as a function of time and accounted for dilution effects or any possible conformational changes likely to occur during dilution.

\section{FITC labeling and characterization of nanoparticles}

Emission spectrum of water-diluted, FITC-labeled nanoparticles was recorded with a fluorescence/luminescence spectrophotometer (Perkin Elmer LS 50B, Beaconsfield, UK) at an excitation wavelength of $485 \mathrm{~nm}$. Further, the fluorescence images of FITC-labeled nanoparticles were observed by confocal laser scanning microscopy (CLSM 510, Carl Zeiss, Jena, Germany). 
A)

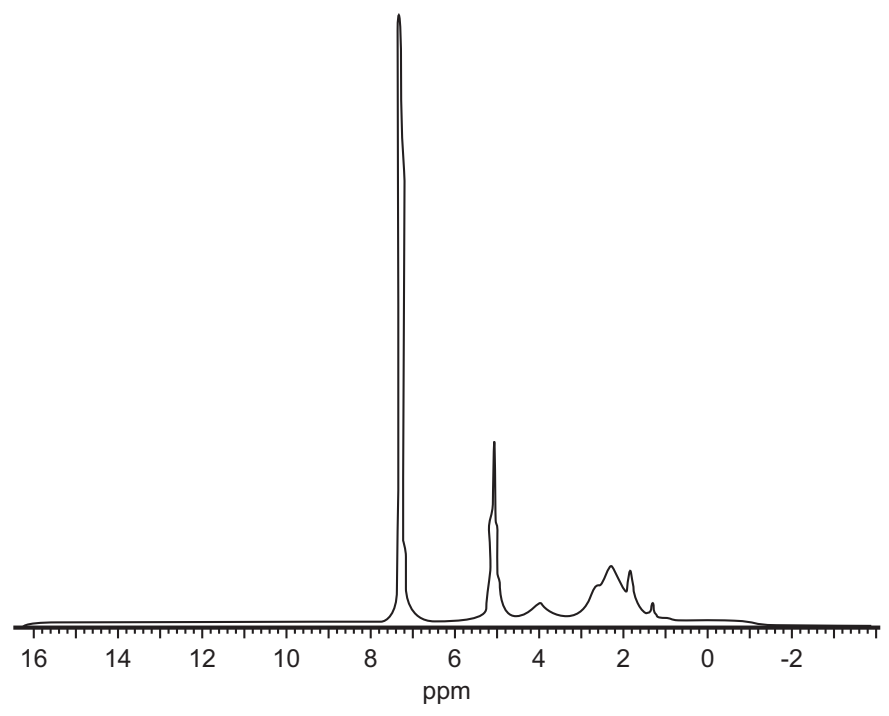

B)

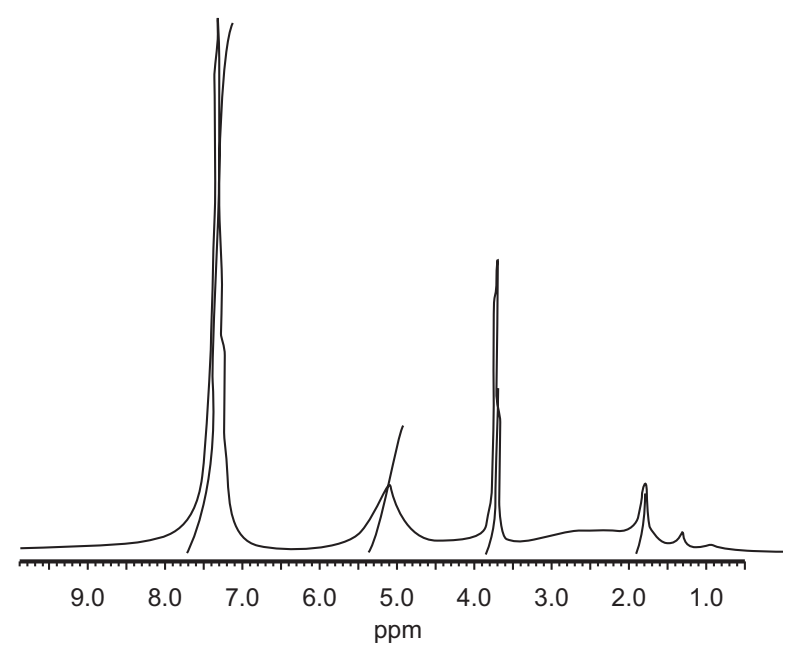

Figure I 'H-NMR spectra of PBLG-Bnz (A) and PBLG-PEG (B).

Abbreviations: Bnz, benzylamine; NMR, nuclear magnetic resonance; PBLG, poly( $\gamma$-benzyl-L-glutamate); PEG, polyethylene glycol.

\section{In vivo studies}

Distribution studies were performed in vivo in Wistar Albino rats (weighing between 250 and $300 \mathrm{~g}$ ) in accordance with an approved specific protocol (Ege University Faculty of Pharmacy Animal Ethics Committee [Protocol no: 2008/6-1]). The animals were divided into three groups (six animals per group), including a control group (G1) and two trial groups (G2 and G3). Prior to the experiments, animals were anesthetized with light ether. Nanoparticles were sterilized by membrane filtration before the in vivo administration. ${ }^{30}$ The saline solution was injected to control group G1, and the two other groups, G2 and G3, received pegylated and nonpegylated nanoparticle suspensions $(0.3 \mathrm{mg} / \mathrm{mL})$, respectively. All animals had free access to food and water ad libitum after the IV injection in the tail vein. Twenty-four hours after administration, the animals were killed by cervical vertebrae dislocation, and their liver and spleen tissues were isolated. All organs were cut and then immediately embedded in Cryoblock (DiaPath, Bergamo, Italy) and cryosectioned $(7 \mu \mathrm{m})$ by cryomicrotome (Leica CM 1900, Wetzlar, Germany) at $-60^{\circ} \mathrm{C}$. Biopsies were spread on microscope glass slides, and analyses were carried out using an Olympus IX71 model fluorescence microscope (Olympus Optical, Tokyo, Japan) equipped with an Olympus DP71 digital camera (Olympus America Corp., Mellville, NY).

\section{Statistical analysis}

Statistical analysis of the data was performed via analysis of variance (ANOVA) followed by Tukey's multiple comparisons test. A $P$ value of less than 0.05 was considered as evidence of a significant difference. ${ }^{31}$ All calculated values were expressed as their mean \pm standard deviation.

\section{Results and discussion Synthesis and characterization of the PBLG derivatives}

Polymerizations were followed by FT-IR spectroscopy. Disappearance of absorption bands at 1843, 1786, and $923 \mathrm{~cm}^{-1}$, corresponding to the cyclic 5-ring anhydrides, indicated the end of the polymerization reaction.

${ }^{1} \mathrm{H}-\mathrm{NMR}$ spectroscopy of synthesized derivates confirmed that the expected structures were obtained for PBLG-Bnz and PBLG-PEG copolymers (Figure 1A, 1B). The signals at $3.72 \mathrm{ppm}$ and $7.36 \mathrm{ppm}$ in the ${ }^{1} \mathrm{H}-\mathrm{NMR}$ spectra were attributed to the PEG backbone and aromatic methylene protons of polymer, respectively (Figure 1B).

High-molecular-weight polymers could be quite easily prepared by ring-opening polymerization in good yields

Table I Molecular weights of synthesized polymers

\begin{tabular}{lll}
\hline Polymer & \multicolumn{2}{l}{ Average molecular weight $(\mathbf{g} / \mathbf{m o l})$} \\
\cline { 2 - 3 } & Theoretical $^{\mathrm{a}}$ & Observed \\
\hline PBLG-Bnz & 50,000 & $46,300^{\mathrm{b}}$ \\
PBLG-PEG & 60,000 & $50,000^{\mathrm{c}}$ \\
PBLG-FITC & 60,000 & $43,300^{\mathrm{b}}$ \\
\hline
\end{tabular}

Notes: ${ }^{2}$ Calculated by considering the constitutional repeating units according to the initial ratio; ${ }^{b}$ Calculated from viscosity measurements; 'Calculated from 'H-NMR spectra.

Abbreviations: Bnz, benzylamine; FITC, fluorescein isothiocyanate; PBLG, poly $(\gamma$ benzyl-L-glutamate); PEG, polyethylene glycol. 
and large quantities. Molecular weights of the synthesized polymers are summarized in Table 1 .

Molecular weights were calculated from viscosity measurements when possible, except for PBLG-PEG copolymers. Molecular weights for PBLG-PEG copolymers were calculated from ${ }^{1} \mathrm{H}$-NMR spectra, because the Mark-Houwink equation was not applicable for these PBLG derivatives. For this copolymer, the degree of polymerization of the PBLG segment in PBLG-PEG derivatives was determined from ${ }^{1} \mathrm{H}-\mathrm{NMR}$ spectra using the ratio between the peak intensities of methylene protons of the PEG chain, $\mathrm{OCH}_{2} \mathrm{CH}_{2}$, and the benzyl protons of the PBLG chain, $\mathrm{COOCH}_{2} \mathrm{C}_{6} \mathrm{H}_{5} .{ }^{1} \mathrm{H}-\mathrm{NMR}$ could not be used for the other PBLG derivatives because the signal from the polymerization initiator was difficult to localize due to the presence of other peaks in the same region.

\section{Physicochemical properties of the nanoparticles}

Stable suspensions of nanoparticles with a moderate polydispersity could be easily and reproducibly prepared from the different synthesized PBLG derivates by a previously described nanoprecipitation method. ${ }^{27}$ Nanoparticles were typically less than $100 \mathrm{~nm}$ and their polydispersity index (PI) was typically less than 0.2 . Further, these particles were negatively charged (zeta potential in the range of $-20 \mathrm{mV}$, which combined with the low PI was in favor of the stability and homogeneity of the suspensions). These characteristics were compatible with an IV administration. ${ }^{7,8}$

As shown in Figure 2, the polymer concentration of the organic phase used during the nanoprecipitation process influenced the mean diameter of the nanoparticles. Concentrations were varied from 0.5 to $2.5 \mathrm{mg} / \mathrm{mL}$ while keeping the other processing parameters at standard conditions. A stronger dependence was observed for nonpegylated nanoparticles

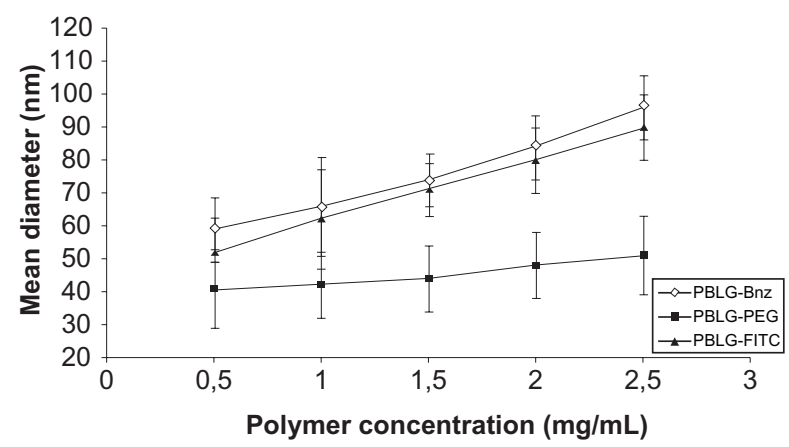

Figure 2 Effect of polymer concentration in the nanoparticle suspension on the mean diameter of nanoparticles.

Abbreviations: Bnz, benzylamine; FITC, fluorescein isothiocyanate; PBLG, poly( $\gamma$-benzyl-L-glutamate); PEG, polyethylene glycol. for which the initial polymer concentration led to a gradual increase in nanoparticle diameter while maintaining a unimodal size distribution (PI values less than 0.2). Under these conditions, the average size of the nanoparticles was significantly increased $(P<0.05)$ from 60 to $100 \mathrm{~nm}$ in the case of PBLG-Bnz and 50 to $90 \mathrm{~nm}$ in the case of PBLGFITC. During the nanoprecipitation process, the mechanisms mastering the self-assembling of amphiphilic polymer chains are quite complex. Commonly, a dependence of the size of the nanoparticles on the viscosity of the organic phase containing the polymer has been observed. For example, the importance of polymer concentration in controlling the size of particles produced by emulsification or nanoprecipitation processes has previously been reported for PLGA and PLA. ${ }^{12,32,33}$ Similar results were found for polycaprolactone/PEG/ polycaprolactone (PCEC) nanoparticles by Huang et al. ${ }^{34}$

Interestingly, very small nanoparticles (circa $40 \mathrm{~nm}$ ) were obtained from the PBLG-PEG copolymer compared with what was obtained with other PBLG derivatives, and almost no influence of the copolymer concentration was observed. These trends could be explained by the amphiphilic nature of this copolymer in comparison with PBLG-Bnz and PBLG-FITC, from which the nanoparticles were prepared without the presence of any surfactant. Indeed, this phenomenon could be explained by the presence of hairy PEG chains $(5000 \mathrm{~g} / \mathrm{mol})$ at the surface of the nanoparticles extending into the water phase. Very small nanoparticles are of interest in targeting applications because the size of the nanoparticles is one of the key characteristics governing their traffic and their distribution in organs and the modalities of their capture by cells. For example, bone targeting requires very small particles, as it is known that the vasculature in bones has pores of approximately $80 \mathrm{~nm}$ in diameter. ${ }^{35}$

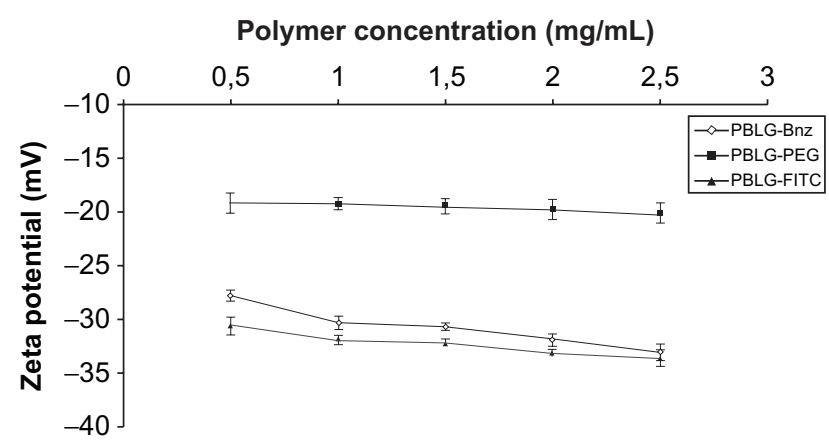

Figure 3 Effect of polymer concentration in the nanoparticle suspension on the zeta potential of the nanoparticles prepared from PBLG-Bnz, PBLG-PEG, and PBLG-FITC. Abbreviations: Bnz, benzylamine; FITC, fluorescein isothiocyanate; PBLG, poly( $\gamma$-benzyl-L-glutamate); PEG, polyethylene glycol. 
Contrary to the particle size, the zeta potential was only slightly changed whatever the nature of the polymer (Figure 3). Pegylated nanoparticles exhibited a negative zeta potential value $(-20 \mathrm{mV})$ closer to neutrality compared with nonpegylated nanoparticles $(-30 \mathrm{mV})$ whatever the initial concentration. This trend is in agreement with other studies describing reductions in the zeta potential following surface pegylation, such as, for example, poly(alkylcyanoacrylate) or polylactide nanoparticles, and could be attributed to the shielding effect of these hydrophilic chains. ${ }^{36,37}$

Finally, storage of these preparations for 4 weeks at $+4^{\circ} \mathrm{C}$ did not show any significant change in nanoparticle characteristics (data not shown), and the suspensions remained stable.

\section{Investigation of protein-nanoparticle interactions by ITC}

Surface pegylation of nanoparticles aims to avoid adsorption of a set of serum proteins such as the complement $\mathrm{C} 3 \mathrm{~b}$ factor following their IV injection (opsonization), which in turn induces their rapid capture by the MPS. ITC analysis was performed to investigate the efficiency of PEG chains to reduce interactions with proteins because of the hydrophilization of the surface of the particles and steric repulsions. As shown earlier by Martinez-Barbosa et al, ${ }^{28}$ ITC can be conveniently used for investigating this effect. In the present study, BSA was used for probing the capacity of PBLG nanoparticles to avoid interactions with proteins.

ITC experiments consist of injecting aliquots of a solution containing a molecule of interest in another solution

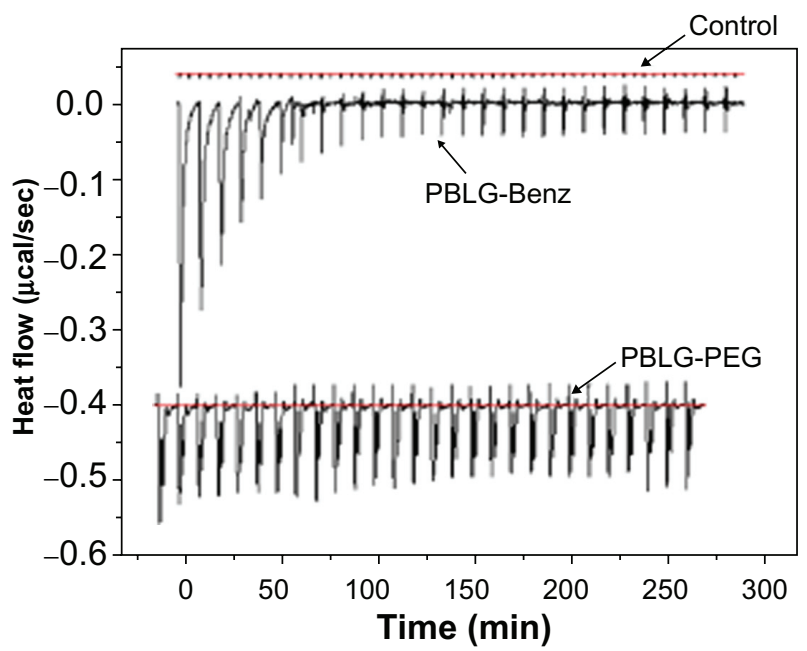

Figure 4 Typical ITC data corresponding to the titration of nonpegylated nanoparticles and pegylated nanoparticles $\left(2.7 \times 10^{-2} \mathrm{mM}\right)$ with BSA solution $\left(5.4 \times 10^{-2} \mathrm{mM}\right)$. Control consisted of the injection of BSA solution into Milli- $\mathrm{Q}^{\circledR}$ water.

Abbreviations: Bnz, benzylamine; BSA, bovine serum albumin; ITC, isothermal titration calorimetry; PBLG, poly( $\gamma$-benzyl-L-glutamate); PEG, polyethylene glycol. containing a second molecule that is supposed to interact, or not, with the first one. Following each injection, heat flow peaks occur that correspond to the amount of heat produced (or consumed) by the interaction developed between the two entities. When the interaction consists of an adsorption phenomenon, a similar behavior is expected. Indeed, as shown in Figure 4, enthalpograms obtained with nonpegylated nanoparticles were different from those obtained with pegylated nanoparticles. Titration of nonpegylated nanoparticles resulted in an initial series of pronounced endothermic peaks, which decreased in intensity after 10 injections and further resulted in fairly constant heat flows when adding BSA. This could correspond to the saturation of the surface in the adsorption process. On the contrary, when the BSA solution was injected into a suspension of pegylated nanoparticles, weaker enthalpy changes were observed than for nonpegylated nanoparticle injection, which could be attributed to decreased interactions of BSA with the surface of the nanoparticles. No significant signal was produced by dilution of the BSA solution in the absence of nanoparticles, which was used as a negative control in this experiment.

\section{FITC labeling and characterizations of optimized nanoparticles}

FITC-labeled nanoparticles were used for in vivo distribution studies. For this purpose, FITC-labeled nanoparticles were prepared from 80:20 (w/w) mixtures of PBLG-Bnz or PBLG-PEG with PBLG-FITC. As shown previously by Martinez-Barbosa et al, ${ }^{27,28}$ the nanoprecipitation of these copolymers leads to the production of composite nanoparticles, each nanoparticle simultaneously containing the two types of polymers. This absence of segregation could be attributed to the similarity of the PBLG segment in these copolymers. Further, it was shown that the incorporation of $77 \%$ of PBLG-PEG copolymer chains in the nanoparticles was enough to maintain the "stealthiness" of the particles, as suggested by ITC adsorption and complementary activation measurements of Martinez-Barbosa et al. ${ }^{28}$ Thus, in the present experiment, an amount of $20 \%$ (w/w) of PBLGFITC was considered to be a good compromise for ensuring stealth properties in the body and simultaneously an optimal fluorescence labeling.

The physicochemical characteristics of these preparations are tabulated in Table 2 . The sizes of pegylated and nonpegylated FITC-labeled nanoparticles were $46 \pm 16 \mathrm{~nm}$ with a PI value of $0.139 \pm 0.01$ and $75 \pm 14 \mathrm{~nm}$ and a narrow size distribution (PI $=0.114 \pm 0.03)$, respectively. In accordance with these results, TEM photographs of the nanoparticles revealed ellipsoi- 
Table 2 Physicochemical properties of FITC-labeled pegylated and nonpegylated nanoparticles $(n=6)$

\begin{tabular}{lllll}
\hline Preparation & $\begin{array}{l}\text { Polymer ratio } \\
(\mathbf{8 0 \%} \text { w/w) }\end{array}$ & $\begin{array}{l}\text { Mean size } \\
(\mathbf{n m})\end{array}$ & PI & $\begin{array}{l}\text { Zeta potential }^{\mathbf{b}} \\
(\mathbf{m V})\end{array}$ \\
\hline Pegylated & PBLG-PEG/PBLG-FITC & $46 \pm 16$ & $0.139 \pm 0.01$ & $-24.5 \pm 0.86$ \\
Nonpegylated & PBLG-Bnz/PBLG-FITC & $75 \pm 14$ & $0.114 \pm 0.03$ & $-29.8 \pm 0.67$ \\
\hline
\end{tabular}

Notes: aMeasured by using a laser light scattering (Nanosize Coulter N4 plus), $n=6$; ' Measured by using a Zetasizer $4, n=6$. Polymer concentration for nanoprecipitation is $1.5 \mathrm{mg} / \mathrm{mL}$.

Abbreviations: Bnz, benzylamine; FITC, fluorescein isothiocyanate; PBLG, poly( $\gamma$-benzyl-L-glutamate); PEG, polyethylene glycol; PI, polydispersity index.

dal nanoparticles with sizes around 50 and $80 \mathrm{~nm}$ for pegylated and nonpegylated formulations, respectively (Figure 5).

Moreover, the FITC labeling efficiency was checked by luminescence spectrophotometer and confocal laser scanning microscopy. ${ }^{38,39}$ The particles emitted green light after excitation at $\lambda=488 \mathrm{~nm}$. Overall, the maximal excitation and emission wavelengths of formulations were found to be $\lambda=486$ and $\lambda=515 \mathrm{~nm}$, respectively, which are close to the values reported for free FITC. ${ }^{40}$

Fluorescence spectra and intensity were also determined in fresh and 4-week-old nanoparticle suspensions stored at $+4^{\circ} \mathrm{C}$ in the dark. No significant differences in their fluorescence spectra or intensities were observed. Overall, maximal excitation and emission wavelengths after storage of the formulations were found to be close to the values reported for free FITC. Such a stability of FITC fluorescence in PBLG nanoparticles has been reported by Segura-Sánchez et al, who have shown that a water suspension of fluorescent nanoparticles has a stable fluorescence after 200-day storage, although free FITC water solutions are not very stable. ${ }^{29}$ This suggests that FITC labeling of PBLG multifunctional nanoparticles is of interest for visualization and tracking purposes.

\section{In vivo studies}

To assess the effect of size and pegylation on the distribution of the nanoparticles, liver and spleen were selected as representative organs of the MPS. ${ }^{15,41,42}$ It is generally

A)

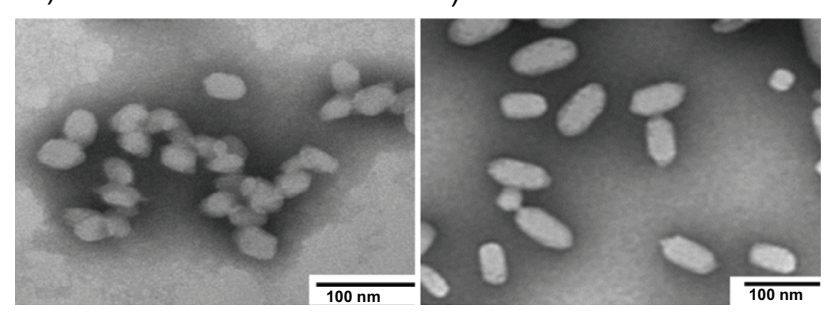

Figure 5 TEM microphotographs of FITC-labeled pegylated $(\mathbf{A})$ and nonpegylated (B) nanoparticles.

Abbreviations: FITC, fluorescein isothiocyanate; TEM, transmission electron microscopy.

accepted that nanoparticles ranging from 30 to $100 \mathrm{~nm}$ in diameter can be captured by the macrophages of the liver and spleen. Liver uptake of the larger particles is faster than for smaller ones. Recent observations indicate that very small particles can pass through the sinusoidal fenestrations in the liver and gain access to the parenchymal cells of the liver. ${ }^{43,44}$ Sonavane et al prepared gold nanoparticles of different particle sizes $(15,50,100$, and $200 \mathrm{~nm})$ and distribution in organs like the liver, lung, and spleen and showed that tissue distribution of gold nanoparticles is size-dependent. ${ }^{45}$ The smallest gold nanoparticles $(15 \mathrm{~nm})$ show the most widespread organ distribution.

The distribution of pegylated and nonpegylated (control) nanoparticles has been evaluated on these tissues in rats. The fluorescence microscopy images of liver and spleen tissue sections are shown in Figures 6 and 7, respectively. Twentyfour hours after IV injection of pegylated nanoparticle suspension (Figures 6C and 7C), only a negligible fluorescence was detected in the liver and spleen when compared with nonpegylated nanoparticles (Figures 6D and 7D). A strong green fluorescence due to the accumulation of nonpegylated nanoparticles could be clearly observed, especially in Kupffer cells, which are located mainly in the periportal region of

A)

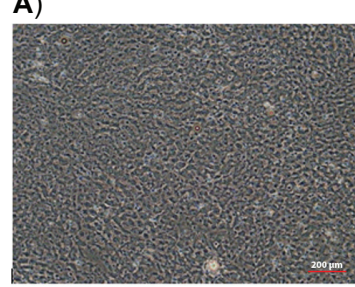

C)

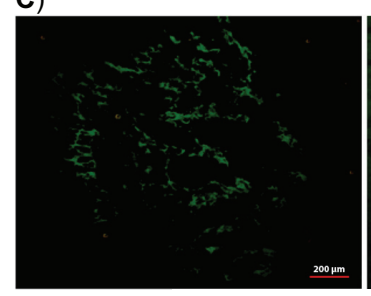

Figure 6 Light image of liver tissue section (A). Fluorescence images of liver tissue sections 24 hours after intravenous injection of: saline (B), pegylated nanoparticles (C), and nonpegylated nanoparticles (D).
B)

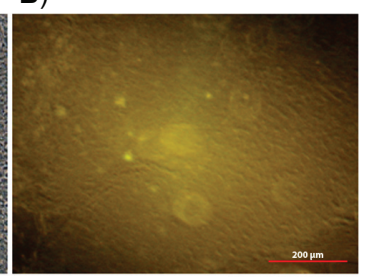

D)

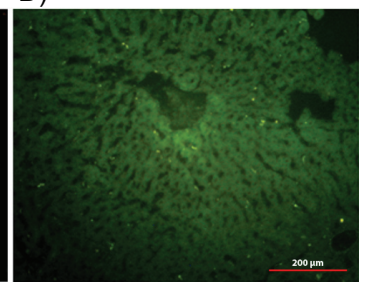


A)

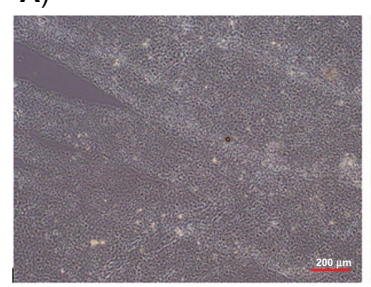

C)

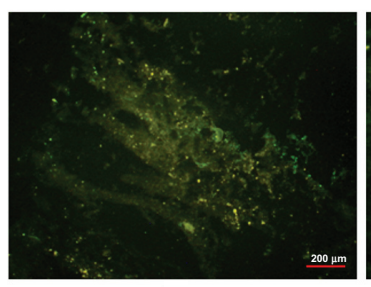

B)

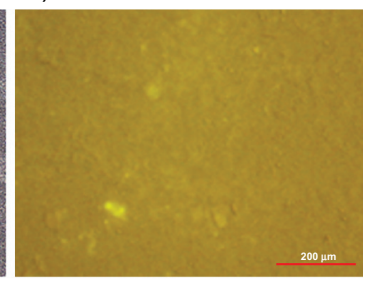

D)

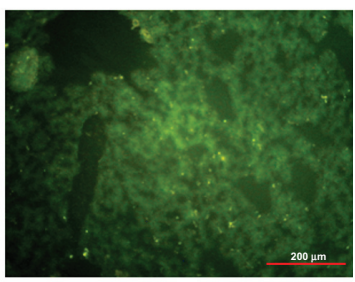

Figure $\mathbf{7}$ Light image of spleen tissue section (A). Fluorescence images of spleen tissue 24 hours after intravenous injection of: saline (B), pegylated nanoparticles (C), and nonpegylated nanoparticles (D).

the liver, and few very bright spots were observed, probably because of aggregation. Green fluorescence along the lining of the liver sinusoids identified endocytosed nanoparticles by liver sinusoidal endothelial cells. On the contrary, the observed weak yellow fluorescence in tissues following injection of pegylated nanoparticles suggests that pegylation of the PBLG nanoparticles was efficient for avoiding capture by the MPS. Similarly, macrophages of the spleen were much less deeply stained after injection of pegylated nanoparticles when compared with nonpegylated nanoparticles (Figure 7C and 7D). No autofluorescence was observed in the animals injected with saline, as expected (Figures 6B and 7B). It is evident from these results that PBLG-PEG copolymers could efficiently self-assemble to create a sufficient concentration of PEG chains at the surface of the particles, which was efficient for avoiding the capture of the PBLG nanoparticles by macrophages.

It can be concluded from this set of experimental data that the outer PEG layer provided an excellent stealth shield for the nanoparticles, while simultaneously the size of the nanoparticles was small enough $(46 \mathrm{~nm})$ to permit the nanoparticles to reach tissues. Indeed, it suggests that essential properties in view of targeted applications can be imparted to composite nanoparticles prepared by auto-assembling of PBLG derivates.

\section{Conclusion}

Due to interesting self-assembling properties, synthetic polypeptides deserve attention as biodegradable materials for the preparation of multifunctional nanoparticles. Belonging to this family, PBLG derivates could be synthesized using ring-opening polymerization, and small nanoparticles with stable characteristics could be successfully prepared by a nanoprecipitation technique without the need for surfactants. Interestingly, small composite pegylated nanoparticles (typically $40 \mathrm{~nm}$ in size) made of a mixture of pegylated and PBLG-FITC copolymers could be obtained. The presence of $80 \%$ of pegylated chains in the nanoparticles was enough to avoid their capture by the MPS, as shown by examination of their biodistribution in the liver and spleen. Because composite PBLG nanoparticles can be looked at as an interesting platform for targeting applications, these preliminary results on the in vivo behavior of these nanoparticles are encouraging and must be considered as the basis for further extensive exploration of the potential of these nanoparticles for targeted drug delivery applications.

\section{Acknowledgments}

We would like to thank the European Frame Socrates/ Erasmus Program for giving us the opportunity to exchange ideas and facilities in conducting this work. This study was also supported by the Research Foundation of Ege University (05/ECZ/014).

\section{Disclosure}

The authors report no conflicts of interest. The authors alone are responsible for the content and writing of the paper.

\section{References}

1. Jeong YI, Seo SJ, Park IK, et al. Cellular recognition of paclitaxel-loaded polymeric nanoparticles composed of poly( $\gamma$-benzyl-L-glutamate) and poly(ethylene glycol) diblock copolymer endcapped with galactose moiety. Int J Pharm. 2005;296:151-161.

2. Martinez-Barbosa ME, Bouteiller L, Cammas-Marion S, Montembault V, Fontaine L, Ponchel G. Synthesis and ITC characterization of novel nanoparticles constituted by poly( $\gamma$-benzyl L-glutamate)- $\beta$-cyclodextrin. J Mol Recognit. 2008;21:169-178.

3. Zhu Z, Li Y, Li X, et al. Paclitaxel-loaded poly(N-vinylpyrrolidone)b-poly( $\varepsilon$-caprolactone) nanoparticles: preparation and antitumor activity in vivo. J Control Release. 2010;142:438-446.

4. Wang LY, Wang SG, Bei JZ. Synthesis and characterization of macroinitiator-amino terminated PEG and poly( $\gamma$-benzyl-Lglutamate)PEO-poly $(\gamma$-benzyl-L-glutamate) triblock copolymer. Polym Adv Technol. 2004;15:617-621.

5. Cheon JB, Jeong YI, Cho CS. Effects of temperature on diblock copolymer micelle composed of poly( $\gamma$-benzyl L-glutamate) and poly(Nisopropylacrylamide). Polymer. 2006;40:2041-2050.

6. Qi L, Xu Z. In vivo antitumor activity of chitosan nanoparticles. Bioorg Med Chem Lett. 2006;16:4243-4245.

7. Geze A, Chau LT, Choisnard L, et al. Biodistribution of intravenously administered amphiphilic $\beta$-cyclodextrin nanospheres. Int J Pharm. 2007;344:135-142.

8. Gaumet M, Vargas A, Gurny R, Delie F. Nanoparticles for drug delivery: The need for precision in reporting particle size parameters. Eur J Pharm Biopharm. 2008;69:1-9.

9. Nanjwade BK, Singh J, Parikh KA, Manvi FV. Preparation and evaluation of carboplatin biodegradable polymeric nanoparticles. Int J Pharm. 2010;385:176-180. 
10. Cheng J, Teply BA, Sherifi I, et al. Formulation of functionalized PLGA-PEG nanoparticles for in vivo targeted drug delivery. Biomater. 2007;28:869-876.

11. Govender T, Riley T, Ehtezazi T, et al. Defining the drug incorporation properties of PLA-PEG nanoparticles. Int J Pharm. 2000;199:95-110.

12. Budhian A, Siegel SJ, Winey KI. Haloperidol-loaded PLGA nanoparticles: Systematic study of particle size and drug content Int J Pharm. 2007;336:367-375.

13. Sepassi S, Goodwin DJ, Drake AF, et al. Effect of polymer molecular weight on the production of drug nanoparticles. J Pharm Sci. 2007;96:2655-2666.

14. Moghimi SM, Szebeni J. Stealth liposomes and long circulating nanoparticles: critical issues in pharmacokinetics, opsonization and protein-binding properties. Prog Lipid Res. 2003;42:463-478.

15. Owens DE, Peppas NA. Opsonization, biodistribution, and pharmacokinetics of polymeric nanoparticles. Int J Pharm. 2006;307:93-102.

16. Decuzzi P, Godin B, Tanaka T, et al. Size and shape effects in the biodistribution of intravascularly injected particles. J Control Release. 2010;141:320-327.

17. Avgoustakis K, Beletsi A, Panagi Z, et al. Effect of copolymer composition on the physicochemical characteristics, in vitro stability, and biodistribution of PLGA-mPEG nanoparticles. Int J Pharm. 2003 259:115-127.

18. Yang T, Cui FD, Choi MK, et al. Enhanced solubility and stability of pegylated liposomal paclitaxel: in vitro and in vivo evaluation. Int J Pharm. 2007;338:317-326.

19. Park J, Fong PM, Lu J, et al. Pegylated PLGA nanoparticles for the improved delivery of doxorubicin. Nanomedicine. 2009;5:410-418.

20. Bhadra D, Bhadra S, Jain P, Jain NK. Pegnology: a review of PEGylated systems. Pharmazie. 2002;57:5-29.

21. Veronese FM, Pasut G. Pegylation, successful approach for drug delivery. Drug Discov Today. 2005;10:1451-1458.

22. Diaz-Lopez R, Tsapis N, Santin M, et al. The performance of pegylated nanocapsules of perfluorooctyl bromide as an ultrasound contrast agent.

23. Gref R, Domb A, Quellec P, et al. The controlled intravenous delivery of drugs using PEG coated sterically stabilized nanospheres. Adv Drug Deliv Rev. 1995;16:215-233.

24. Zhen XL, Kan B, Gou ML, et al. Preparation of mPEG-PLA nanoparticle for honokiol delivery in vitro. Int J Pharm. 2010;386:262-267.

25. Redhead HM, Davis SS, Illum L. Drug delivery in poly(lactideco-glycolide) nanoparticles surface modified with poloxamer 407 and poloxamine 908: in vitro characterisation and in vivo evaluation. J Control Release. 2001;70:353-363.

26. Zambaux MF, Faivre-Fiorina B, Bonneaux F, et al. Involvement of neutrophilic granulocytes in the uptake of biodegradable non-stealth and stealth nanoparticles in guinea pig. Biomaterials. 2000;21:975-980.

27. Martinez-Barbosa ME, Montembault V, Cammas-Marion S, Ponchel G, Fontaine L. Synthesis and characterization of novel poly $(\gamma$-benzyl-Lglutamate) derivatives tailored for the preparation of nanoparticles of pharmaceutical interest. Polym Int. 2007;56:317-324.

28. Martinez-Barbosa ME, Cammas-Marion S, Bouteiller L, Vauthier C, Ponchel G. Pegylated degradable composite nanoparticles based on mixtures of PEG- $b$-poly ( $\gamma$-benzyl L-glutamate) and poly $(\gamma$-benzyl L-glutamate). Bioconjugate Chem. 2009;20:1490-1496. Biomater. 2010;31:1723-1731.

29. Segura-Sánchez F, Montembault V, Fontaine L, Martínez-Barbosa E, Bouchemal K, Ponchel G. Synthesis and characterization of functionalized poly $(\gamma$-benzyl-L-glutamate) derivates and corresponding nanoparticles preparation and characterization. Int J Pharm. 2010; $387: 244-252$

30. Ozcan I, Segura-Sánchez F, Bouchemal K, et al. Effects of sterilization techniques on the PEGylated poly $(\gamma$-benzyl-L-glutamate) (PBLG) nanoparticles. Acta Pharm Sciencia. 2009;51:211-218.

31. Dawson B, Trapp RG. Basic and Clinical Biostatistics. USA: McGraw Hill; 2001:161-182.

32. $\mathrm{Mu} \mathrm{L}$, Feng SS. A novel controlled release formulation for the anticancer drug paclitaxel $\left(\right.$ Taxol $\left.^{\circledR}\right)$ : PLGA nanoparticles containing vitamin E TPGS. J Control Release. 2003;86:33-48.

33. Song X, Zhao Y, Wu W, et al. PLGA nanoparticles simultaneously loaded with vincristine sulfate and verapamil hydrochloride: systematic study of particle size and drug entrapment efficiency. Int J Pharm. 2008;350:320-329.

34. Huang MJ, Gou ML, Qian ZY, et al. One-step preparation of poly(epsiloncaprolactone)- poly(ethylene glycol)-poly(epsiloncaprolactone) nanoparticles for plasmid DNA delivery. J Biomed Mater Res A. 2008;86:979-986.

35. Wang D, Miller S, Sima M, Kopeckova P, Kopecek J. Synthesis and evaluation of water-soluble polymeric bone-targeted drug delivery systems. Bioconjugate Chem. 2003;14:853-859.

36. Peracchia MT, Fattal E, Desmaele D, et al. Stealth pegylated polycyanoacrylate nanoparticles for intravenous administration and splenic targeting. J Control Release. 1999;60:121-128.

37. Vila A, Gill H, McCallion O, Alonso MJ. Transport of PLA-PEG particles across the nasal mucosa: effect of particle size and PEG coating density. J Control Release. 2004;98:231-244.

38. Kim TH, Park TG. Critical effect of freezing/freeze-drying on sustained release of FITC-dextran encapsulated within PLGA microspheres. Int J Pharm. 2004;271:207-214.

39. Pygall SR, Whetstone J, Timmins P, Melia CD. Pharmaceutical applications of confocal laser scanning microscopy: The physical characterisation of pharmaceutical systems. Adv Drug Del Rev. 2007;59: 1434-1452.

40. Zhao J, Wu J. Preparation and characterization of the fluorescent chitosan nanoparticle probe. Chinese J Anal Chem. 2006;34:1555-1559.

41. Guo G, Liu W, Liang J, Xu H, He Z, Yang X. Preparation and characterization of novel CdSe quantum dots modified with poly (D, L-lactide) nanoparticles. Mater Lett. 2006;60:2565-2568.

42. Suh J, Choy KL, Lai SK, et al. PEGylation of nanoparticles improves their cytoplasmic transport. Int J Nanomed. 2007;2:735-741.

43. Stolnik S, Heald CR, Neal J, et al. Polylactide-poly(ethylene glycol) micellar-like particles as potential drug carriers: production, colloidal properties and biological performance. J Drug Target. 2001;9: 361-378.

44. Beletsi A, Panagi Z, Avgoustakis K. Biodistribution properties of nanoparticles based on mixtures of PLGA with PLGA-PEG diblock copolymers. Int J Pharm. 2005;298:233-241.

45. Sonavane G, Tomoda K, Makino K. Biodistribution of colloidal gold nanoparticles after intravenous administration: effect of particle size. Colloids Surf B Biointerfaces. 2008;66:274-280.

International Journal of Nanomedicine

\section{Publish your work in this journal}

The International Journal of Nanomedicine is an international, peerreviewed journal focusing on the application of nanotechnology in diagnostics, therapeutics, and drug delivery systems throughout the biomedical field. This journal is indexed on PubMed Central,

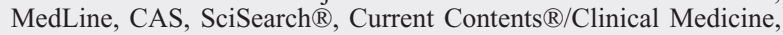

\section{Dovepress}

Journal Citation Reports/Science Edition, EMBase, Scopus and the Elsevier Bibliographic databases. The manuscript management system is completely online and includes a very quick and fair peer-review system, which is all easy to use. Visit http://www.dovepress.com/ testimonials.php to read real quotes from published authors. 